\title{
COMPARAÇÃO DE ROTAS CONSOLIDADAS PARA A PRODUÇÃO DE BIODIESEL A PARTIR DOS ÓLEOS DE SOJA E DE DENDÊ VIA CATÁLISE HOMOGÊNEA ALCALINA
}

\author{
A. F. YOUNG ${ }^{1}$, F. L. P. PESSOA ${ }^{2}$ e E. M. QUEIROZ ${ }^{2}$ \\ ${ }^{1}$ Universidade Federal do Rio de Janeiro, Escola de Química \\ ${ }^{2}$ Universidade Federal do Rio de Janeiro, Escola de Química, Departamento de Engenharia \\ Química \\ E-mail para contato: andreyoung@ufrj.br
}

\begin{abstract}
RESUMO - Foi simulada a produção de biodiesel a partir do óleo de soja e de dendê a nível industrial. As propriedades termodinâmicas mais importantes dos componentes foram estimadas pelo método de Constantinou e Gani. Foi mostrado que algumas alterações nas correntes de entrada do processo e nos equipamentos são necessárias se objetiva-se processar tanto o óleo de soja quanto o de dendê, a fím de gerar um combustível que atenda às principais normas nacionais e internacionais de qualidade.
\end{abstract}

\section{INTRODUÇÃO}

Nos últimos anos, o aumento da preocupação ambiental aliado à diminuição progressiva das reservas de petróleo mundiais tem motivado o estudo e desenvolvimento de tecnologias e fontes de energia sustentáveis. Nesse contexto, surgem os biocombustíveis e, dentre eles, o biodiesel.

O biodiesel é uma alternativa ao diesel tradicional. É um combustível renovável, produzido a partir de óleos vegetais ou gordura animal através da transesterificação desses componentes com um álcool, normalmente metanol ou etanol. É biodegradável, atóxico e a sua queima emite menos poluentes do que a queima do diesel produzido a partir do petróleo (Meher et al., 2006).

Com relação a matéria prima, os óleos e gorduras são constituídos por triglicerídeos, ésteres de glicerol e ácidos graxos. Os óleos vegetais podem ser obtidos de diversas fontes. Tratando-se de Brasil, dá-se especial atenção à soja, que é capaz de gerar de 400 a 650 litros de óleo por hectare. No entanto, é crescente no norte, nordeste e centro-oeste do país a utilização da palma (ou dendê), por sua elevada popularidade e produção de 5.500 a 8.000 litros de óleo por hectare (Oliveira et al., 2008).

A produção do biodiesel começa com o tratamento do óleo. Este deve passar por processos de neutralização, para redução da acidez, e desumidificação. A matéria prima tratada segue para um tanque, onde é misturada com o álcool escolhido e o catalisador (se necessário). São conhecidas diversas vias de produção de biodiesel, dentre as quais destacam-se a catálise homogênea ácida, a catálise homogênea alcalina (sendo essa a mais comum), a catálise heterogênea, a pirólise, a catálise enzimática e o processamento com fluidos supercríticos (Oliveira et al., 2008; Innocentini, 


\section{9 a 22 de outubro de 2014 \\ Florianópolis/SC}

2007; Cubas, 2010).

O objetivo do trabalho é comparar, com base em fluxogramas básicos de processos, o desempenho da catálise alcalina homogênea para a obtenção de biodiesel a partir do óleo de soja, o mais utilizado no Brasil, e de dendê, interessante por conter um teor maior de ácidos graxos livres e possuir uma relação de produção de óleo por hectare bastante superior.

\section{ESTADO DA ARTE}

Dentre as tecnologias para produção de biodiesel, definitivamente a mais bem consolidada é a catálise alcalina e muitos estudos foram feitos na tentativa de implementa-la a nível industrial. Kreutzer (1984) sugeriu um processo em altas pressões (90 bar) e temperaturas $\left(240{ }^{\circ} \mathrm{C}\right)$, mas críticas foram feitas a ele por conta do alto custo dos equipamentos envolvidos, do alto consumo de energia e dos problemas com segurança. Na sequência, outros autores continuaram desenvolvendo soluções para essas questões até que Connemann e Fischer (1998) estruturaram um processo para produção de biodiesel por catálise alcalina a pressão ambiente e temperaturas entre 65 e $70{ }^{\circ} \mathrm{C}$. Esse processo teve imediato sucesso e é hoje a base para o desenvolvimento de novos fluxogramas. Ele faz uso de uma coluna de destilação para separar o metanol do biodiesel e do glicerol. O metanol é reciclado para o reator de transesterificação e a purificação do biodiesel é feita através de lavagem com água.

Uma limitação do processo de produção do biodiesel por catálise alcalina é a sua sensibilidade à umidade e acidez da matéria prima vegetal. Por conta disso, foram feitos estudos visando o desenvolvimento de pré-tratamentos para o óleo vegetal. Lepper e Friesenhagen (1986) recomendaram um pré-tratamento baseado na esterificação dos ácidos graxos livres no óleo vegetal com metanol na presença de ácido sulfúrico. Esse tipo de pré-tratamento parece ser ainda hoje o mais adotado em trabalhos de simulação de processos.

Zhang et al. (2003 a,b) e West et al. (2007) compararam diferentes rotas de produção de biodiesel através de simuladores de processo. Foram avaliados os fluxos materiais e de energia e dimensionados os equipamentos, de modo a avaliar cada processo economicamente. Foi demonstrado que os preços do óleo vegetal e do biodiesel e a capacidade da planta foram os fatores que mais afetaram o custo total de produção. Esses trabalhos formam a base dos estudos de simulação de processos que vieram em seguida e foram também a base para este trabalho.

\section{SIMULAÇÃO DO PROCESSO}

O processo de transesterificação do óleo de soja e de dendê via catálise homogênea alcalina foi simulado no software PRO/II $9.1 ~ \circledR$, desenvolvido pela Invensys Systems, Inc. O procedimento para a simulação de processos envolve a definição dos componentes químicos presentes, a seleção do pacote termodinâmico, a determinação da capacidade de produção, a seleção dos equipamentos necessários e a montagem do fluxograma de processo.

\subsection{Definição dos Componentes Químicos}

O álcool escolhido para a transesterificação foi o etanol, por causa de sua grande disponibilidade em território brasileiro. Apesar de mais caro, de ser mais difícil de separar e de 


\section{9 a 22 de outubro de 2014 \\ Florianópolis/SC}

exigir maiores custos de energia e equipamentos, o etanol é menos tóxico, menos volátil, tem um menor risco de incêndio, produz um biodiesel com maior índice de cetano e lubricidade, além de ser inteiramente renovável (Quelhas, 2012).

Além do etanol, os outros componentes presentes nas simulações do óleo de soja e de dendê são glicerol, água, hidróxido de sódio, ácido sulfúrico e sulfato de sódio. Hidróxido de sódio foi escolhido como o catalisador da reação de transesterificação por conta de seu baixo preço e elevada eficiência. O mesmo critério foi utilizado para a escolha do ácido sulfúrico como catalisador da reação de esterificação no pré-tratamento da matéria-prima. Outras opções seriam hidróxido de potássio e ácido fosfórico, por exemplo.

Todos os componentes descritos anteriormente encontram-se disponíveis na base de dados do simulador. No intuito de melhor representar as peculiaridades de cada um dos óleos vegetais e seus respectivos produtos, óleo de soja, óleo de dendê, ácidos graxos livres da soja, ácidos graxos livres do dendê, biodiesel de soja e biodiesel de dendê foram tratados como seis pseudocomponentes, os quais tiveram as suas propriedades estimadas e inseridas no programa.

Cavalcante (2010) estimou as propriedades críticas de biodiesel proveniente de diversas matérias-primas. Dentre os métodos utilizados, o método de contribuição de grupos de Constantinou e Gani foi o que apresentou os melhores resultados. Esse foi o método utilizado nesse trabalho, também por ser um método simples, completo, relativamente recente, além de não sofrer contraindicações para a modelagem de ácidos graxos (Poling et al., 2004). Constantinou e Gani desenvolveram um avançado método de contribuição de grupos baseado nos grupos do modelo termodinâmico UNIFAC. Neste trabalho, foram utilizados apenas os grupos de primeira ordem. Para o cálculo do fator acêntrico, foi utilizada a regra de mistura de Lee-Kesler (Reid et al., 1987).

As propriedades dos óleos, combustíveis e ácidos graxos são mostradas na Tabela 1.

Tabela 1- Propriedades termodinâmicas de óleos, biodiesel e ácidos graxos

\begin{tabular}{|c|c|c|c|c|c|c|}
\hline & Óleo de Soja & Óleo de Dendê & Biodiesel de Soja & Biodiesel de Dendê & Ácidos da Soja & Ácidos do Dendê \\
\hline $\mathrm{Tc}(\mathrm{K})$ & 976,675 & 969,040 & 781,387 & 773,905 & 796,760 & 789,925 \\
\hline $\mathrm{Pc}(\mathrm{Kpa})$ & 648,416 & 655,010 & 1259,137 & 1274,218 & 1419,857 & 1444,414 \\
$\mathrm{~Tb}(\mathrm{~K})$ & 835,981 & 828,670 & 621,469 & 614,510 & 625,481 & 618,675 \\
$\mathrm{Vc}(\mathrm{m} 3 / \mathrm{kmol})$ & 3,195 & 3,115 & 1,145 & 1,119 & 1,033 & 1,006 \\
W & 0,416 & 0,438 & 0,806 & 0,811 & 0,813 & 0,814 \\
\hline
\end{tabular}

\subsection{Definição do Pacote Termodinâmico}

Devido à presença de compostos fortemente polares, como o etanol e o glicerol, o pacote termodinâmico adotado para os cálculos dos coeficientes de atividade de cada componente foi o non-random two liquid (NRTL), conforme proposto por Zhang et al. (2003 a). Os parâmetros de interação binária que não estavam presentes na base de dados do simulador foram estimados com base nas estruturas UNIFAC dos compostos. Para os óleos, ácidos graxos e biodiesel, as estruturas UNIFAC adotadas foram as dos componentes palmitato para o dendê e linoleato para a soja.

\subsection{Determinação da Capacidade de Produção}

De acordo com o Boletim Mensal do Biodiesel de novembro de 2013, publicado pela Agência Nacional do Petróleo, Gás Natural e Biocombustíveis (ANP), a produção total de 
biodiesel em território brasileiro era de 22.619,06 m³/dia. Eram, então, 67 plantas autorizadas, correspondendo a uma produção média de $14,07 \mathrm{~m}^{3} / \mathrm{h}$ de biodiesel. Considerando a densidade média a temperatura ambiente calculada por Cavalcante (2010) para o biodiesel de soja, principal tipo de biodiesel no Brasil, a capacidade mássica média das unidades brasileiras correspondia a $12.283 \mathrm{Kg} / \mathrm{h}$. Essa foi, portanto, a capacidade escolhida para o presente trabalho.

\subsection{Montagem do Fluxograma de Processo}

Optou-se por iniciar o processo com uma etapa inicial de pré-tratamento para a remoção de acidez do óleo vegetal. Embora seja normalmente atrelada à fase de refino do óleo vegetal, essa etapa é de suma relevância para a comparação entre as duas fontes de matéria-prima, dado que o óleo de dendê apresenta uma acidez em média dez vezes superior à do óleo de soja. Portanto, as matérias-primas consideradas no trabalho são os óleos brutos de soja e de dendê.

A etapa de pré-tratamento é mostrada na Figura 1 e é baseada no trabalho de Lepper e Friesenhagen (1986). O óleo vegetal é alimentado a um reator de mistura. Etanol, $15 \%$ do volume da fase óleo, é previamente misturado com o catalisador ácido, $1 \%$ da massa de óleo, e adicionado ao reator. A reação se processa a $70{ }^{\circ} \mathrm{C}$ e $400 \mathrm{KPa}$. Como não se tem informações suficientes acerca da cinética de esterificação dos ácidos graxos envolvidos, considerou-se uma conversão de $95 \%$. Após a reação, o óleo refinado segue para uma coluna de lavagem com glicerina, para remoção do ácido sulfúrico e água, que têm que ser retirados completamente antes da transesterificação. A lavagem com glicerina foi simulada numa coluna de extração com quatro pratos teóricos. A glicerina é alimentada no topo da coluna a $25^{\circ} \mathrm{C}$ e $200 \mathrm{KPa}$. Finalmente, a corrente extrato proveniente da coluna de extração é alimentada a uma coluna de destilação de cinco estágios teóricos e razão de refluxo igual a cinco para a separação do etanol. O objetivo dessa coluna é propiciar que, tanto o etanol liberado no topo da coluna quanto a glicerina obtida no fundo saiam em condições de serem reutilizados no processo. A corrente rafinado, proveniente da coluna de extração, é bombeada e aquecida e segue para a etapa de transesterificação.

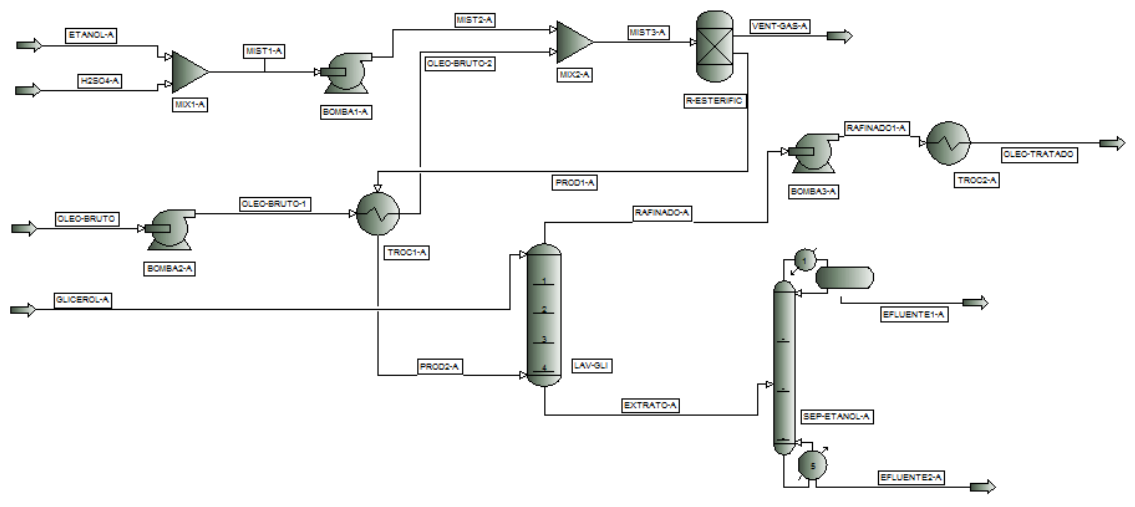

Figura 1 - Fluxograma da etapa de pré-tratamento.

A etapa de transesterificação é mostrada na Figura 2. Novamente, etanol é previamente misturado com o catalisador, agora o hidróxido de sódio, antes de ser adicionado ao reator. Foi utilizada uma razão 9:1 molar de etanol com relação ao óleo e $1 \%$ de catalisador. A reação se 
processa em um reator de mistura, a $50{ }^{\circ} \mathrm{C}$ e $400 \mathrm{KPa}$. Novamente, por não haver informação suficiente com relação a cinética da reação de transesterificação, foi assumida uma conversão de $95 \%$, que está de acordo com o trabalho de Garnica (2009). Também foi a conversão assumida por Zhang et al. (2003 a) e West et al. (2008) em seus trabalhos, em condições próprias para a reação com metanol, no caso.

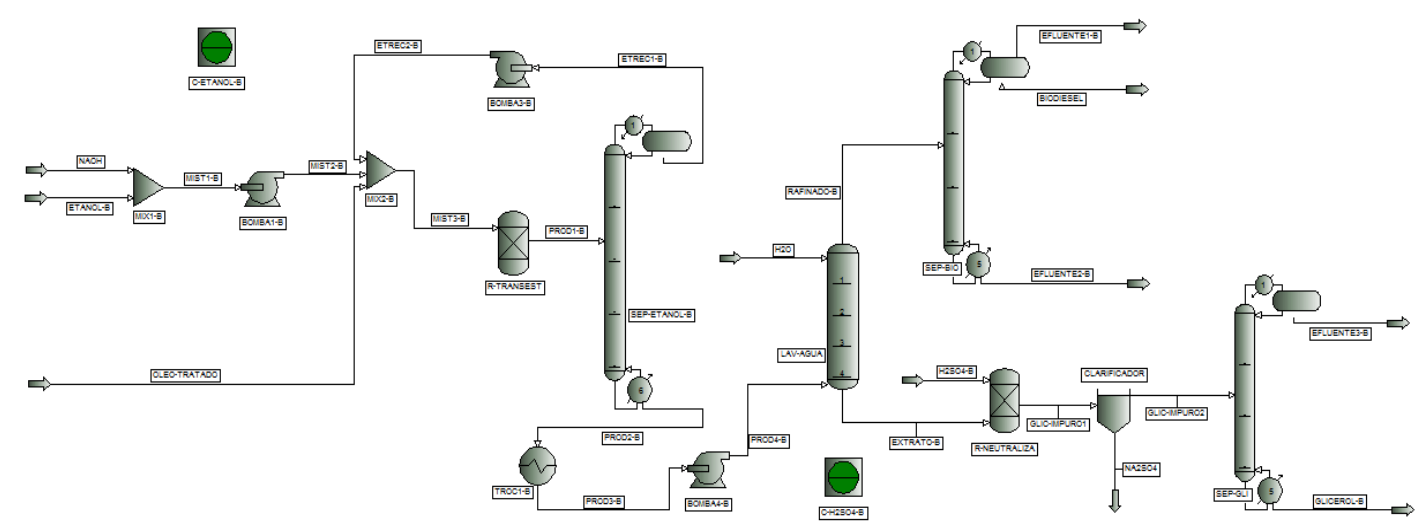

Figura 2 - Fluxograma da etapa de transesterificação.

O produto da reação é diretamente alimentado a uma coluna de destilação de seis estágios teóricos e razão de refluxo igual a dois, responsável por reciclar o excesso de etanol de volta para o reator. A vazão da corrente de reciclo, bem como a entrada de etanol na planta, são ajustadas pelo controlador C-Etanol-B para garantir que a razão de 9:1 molar etanol-óleo seja mantida.

$\mathrm{O}$ produto de fundo da coluna é resfriado e segue para lavagem. A lavagem é feita com água, a cerca de $60{ }^{\circ} \mathrm{C}$ e $110 \mathrm{KPa}$, numa coluna de extração com quatro estágios teóricos. O rafinado segue, então, para a coluna de purificação do biodiesel. Já o extrato, rico em glicerol, segue para as etapas de purificação do mesmo.

A primeira etapa na linha de purificação do glicerol é a neutralização do hidróxido de sódio do meio com a adição de ácido sulfúrico em proporções estequiométricas. Isso é feito em um reator de mistura a $60{ }^{\circ} \mathrm{C}$ e pressão atmosférica. Nessas condições, foi considerada conversão total do hidróxido de sódio. O produto é levado, então, a um tanque clarificador, onde o sulfato de sódio é removido por ação gravitacional.

O glicerol, ainda impuro, segue para uma coluna de destilação, onde é separado tanto quanto possível do etanol e da água, em cinco pratos teóricos e razão de refluxo igual a um (em base molar). A coluna opera sob vácuo para evitar a degradação do glicerol.

O biodiesel que sai da coluna de extração ainda contém um elevado teor de álcool, água e óleo não reagido. Essa mistura é alimentada numa coluna de destilação de quatro ou cinco pratos teóricos e razão de refluxo igual a dois para purificação do biodiesel. Uma mistura rica em óleo e biodiesel é retirada no fundo. No topo, é retirada uma mistura de etanol e água. A coluna, tal qual 


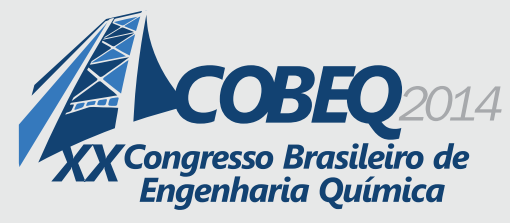

as demais, é operada sob vácuo, para que as temperaturas de topo e de fundo não atinjam a temperatura de degradação do biodiesel.

\section{RESULTADOS E DISCUSSÃO}

As Tabelas 2 e 3 mostram os resultados para as correntes de entrada e saída das plantas de soja e dendê, respectivamente. Na composição da corrente Óleo Bruto fica clara a diferença de acidez entre os dois óleos. No entanto, como a quantidade de etanol na esterificação foi regulada de acordo com a vazão volumétrica de entrada de óleo e a densidade de óleo e ácidos graxos é semelhante, a quantidade de etanol necessário para a conversão dos ácidos graxos foi bastante próxima para a soja e o dendê. Diferenças pequenas também foram observadas nas entradas de glicerol e água necessários para as lavagens.

Tabela 2 - Propriedades das correntes de entrada e saída do processo para o óleo de soja

\begin{tabular}{|c|c|c|c|c|c|c|c|c|c|c|c|c|}
\hline Nome da Corrente & Etanol-A & Óleo Bruto & Glicerol-A & Efluente1-A & Efluente2-A & Etanol-B & $\mathrm{H}_{2} \mathrm{O}$ & Efluente1-B & Efluente2-B & Biodiesel & Efluente3-B & Glicerol-B \\
\hline Temperatura $\left({ }^{\circ} \mathrm{C}\right)$ & 25,0000 & 25,0000 & 25,0000 & 42,0555 & 135,1611 & 25,0000 & 25,0000 & 176,0224 & 300,0000 & 176,0224 & 59,3742 & 81,9810 \\
\hline Pressão (Kpa) & 101,3000 & 101,3000 & 101,3000 & 20,0000 & 30,0000 & 101,3000 & 101,3000 & 10,0000 & 20,0000 & 10,0000 & 40,0000 & 50,0000 \\
\hline Vazão mássica (Kg/h) & 1648,3406 & 12717,7597 & 2325,5332 & 1526,7638 & 2477,7787 & 2417,0965 & 290,6917 & 448,8661 & 954,0086 & 12283,5288 & 584,6928 & 1276,0695 \\
\hline \multicolumn{13}{|c|}{ Vazão mássica por componente } \\
\hline Óleo de Soja & 0,0000 & 12654,1709 & 0,0000 & 0,0000 & 0,0014 & 0,0000 & 0,0000 & 0,0000 & 632,3283 & 0,3801 & 0,0000 & 0,0000 \\
\hline Biodiesel & 0,0000 & 0,0000 & 0,0000 & 0,0000 & 0,1084 & 0,0000 & 0,0000 & 123,7141 & 321,5266 & 12277,0341 & 0,5682 & 0,0028 \\
\hline Ácidos Gr & 0000 & 63,5888 & 0000 & 0,0000 & ,0123 & 0,0000 & 0,0000 & 0,0266 & 0,1051 & ,0349 & 0,0000 & 0,0006 \\
\hline Glicerol & 000 & & 2325 & 00 & 231 & 00 & & & 00 & 000 & 0,8104 & 1270,9245 \\
\hline Etanol & 1648,3406 & 0,0000 & 0,0000 & 1522,9460 & 31,0805 & 2417,0965 & 0,0000 & 274,5753 & 0,0000 & .7735 & 291,2478 & 0,1109 \\
\hline $\mathrm{H}_{2} \mathrm{O}$ & 0,0000 & 0,0000 & 0,0000 & 3,8178 & 0,0839 & 0,0000 & 290,6917 & 50,5501 &, 0000 & 3057 & 292,0664 & ,0302 \\
\hline $\mathrm{H}_{2} \mathrm{SO}$ & 0,0000 & 0,0000 & 00 & 0,0000 & 127,1772 & 0,0000 & 0,0000 & , 0000 & 0,0000 & 0000 & 0,0000 & ,0005 \\
\hline $\mathrm{NaOH}$ & 0,0000 & 0,0000 & 00 & 0,0000 & 000 & 0,0000 & 0,0000 & 000 & 0,0486 & 0005 & 0,0000 & 0,0000 \\
\hline $\mathrm{Na}_{2} \mathrm{SO}_{4}$ & 0,0000 & 0,0000 & 0,0000 & 0,0000 & 0,0000 & 0,0000 & 0,0000 & 0,0000 & 0,0000 & 0,0000 & 0,0000 & 0,0000 \\
\hline
\end{tabular}

Uma diferença um pouco maior foi observada em relação às vazões de entrada de etanol na transesterificação. A quantidade necessária para o dendê é menor, pois o óleo possui um teor menor de triglicerídeos. O resultado é um biodiesel de dendê com um maior teor de acidez que o biodiesel de soja. Essa diferença com certeza implicará em diferentes propriedades entre os combustíveis. No entanto, é importante destacar que ambos os combustíveis obtidos neste trabalho se encontram dentro das normas brasileira (ANP 05/2012), europeia (EN14214) e americana (ASTM D6751) no que diz respeito à teor de ésteres, teor de água, índice de acidez, glicerol livre, teor de triglicerídeos e teor de álcool.

São observadas cinco correntes efluentes do processo. A corrente Efluente1-A deixa o topo da coluna Sep-Etanol-A e é composta por etanol e água. Uma possível solução para essa corrente é a separação da maior quantidade possível de água e reutilização do etanol na etapa de esterificação. A corrente Efluente2-A deixa o fundo da mesma coluna. Ela é composta majoritariamente por glicerol, mas devido ao seu alto índice de acidez, não pode ser reciclada diretamente para o processo. Ela poderia ser tratada para ser reutilizada na lavagem com glicerol. A corrente Efluente1-B deixa o topo da coluna de purificação do biodiesel. Seu destino pode ser o mesmo da corrente Efluente-1-A, dado que sua utilização na transesterificação não é indicada por conta da presença de água. A corrente Efluente2-B representa o óleo não-processado, que deixa o processo com quantidades consideráveis de biodiesel e traços de ácidos graxos e hidróxido de sódio cuja maior tendência é reagirem formando sabão. Maior atenção deve ser dada ao tratamento dessa corrente, tendo em vista a preservação ambiental. E a corrente Efluente3-B é mais uma corrente rica em etanol e água que, por simplificação processual, pode receber o mesmo destino das correntes Efluente1-A e Efluente1-B. A separação dessa corrente serve para produção 


\section{9 a 22 de outubro de 2014 \\ Florianópolis/SC}

de glicerina com qualidade suficiente para a venda, agregando valor à produção de biodiesel e reduzindo um dos principais rejeitos dessa indústria.

Tabela 3 - Propriedades das correntes de entrada e saída do processo para o óleo de dendê

\begin{tabular}{|c|c|c|c|c|c|c|c|c|c|c|c|c|}
\hline Nome da Corrente & Etanol-A & Óleo Bruto & Glicerol-A & Efluente1-A & Efluente2-A & Etanol-B & $\mathrm{H}_{2} \mathrm{O}$ & Efluente1-B & Efluente2-B & Biodiesel & Efluente3-B & Glicerol-B \\
\hline Temperatura $\left({ }^{\circ} \mathrm{C}\right)$ & 25,0000 & 25,0000 & 25,0000 & 42,0956 & 111,9753 & 25,0000 & 25,0000 & 173,5279 & 300,0000 & 173,5279 & 58,3955 & 81,9647 \\
\hline Pressão (Kpa) & 101,3000 & 101,3000 & 101,3000 & 20,0000 & 30,0000 & 101,3000 & 101,3000 & 10,0000 & 20,0000 & 10,0000 & 40,0000 & 50,0000 \\
\hline Vazão mássica $(\mathrm{Kg} / \mathrm{h})$ & 1628,9605 & 12556,3311 & 2260,1600 & 1416,7102 & 2406,7924 & 2252,2493 & 275,0551 & 490,6335 & 807,2128 & 12282,3911 & 532,7848 & 1249,8386 \\
\hline \multicolumn{13}{|c|}{ Vazão mássica por comp onent } \\
\hline Óleo de Dendê & 0,0000 & 11928,5145 & 0,0000 & 0,0000 & 0,0050 & 0,0000 & 0,0000 & 0,0000 & 596,0978 & 0,3277 & 0,0000 & 0,0000 \\
\hline Biodiesel & 0,0000 & 0,0000 & 0,0000 & 0,0000 & 471 & 0000 & 0,0000 & 144,3303 & 210,2876 & 12248,7514 & 0,3061 & 0,5036 \\
\hline Ácidos Graxos & 0,0000 & 627,8166 & 0,0000 & 0,0000 & 0,1446 & 0,0000 & 0,0000 & 73 & 0,7731 & 30,1592 & 0,0000 & 0,0065 \\
\hline Glicerol & 0,0000 & 0,0000 & 2260,1600 & 0,0000 & 2251,4313 & 0,0000 & 0,0000 & 0,0000 & 0,0000 & 0,0000 & 0,3714 & 1237,3403 \\
\hline Etanol & 1628,9605 & 0,0000 & 0,0000 & 1376,9701 & 28,1014 & 2252,2493 & 0,0000 & 296,0550 & 0,0000 & 2,9905 & 262,4418 & 0,1702 \\
\hline 0 & 0000 & 0,0000 & 0 & 39,7401 & .0002 & 0,0000 & 275,0551 & 19 & 00 & 23 & 269,6655 & 11,8175 \\
\hline $\mathrm{H}_{2} \mathrm{SO}_{4}$ & 0,0000 & 0,0000 & 0,0000 & 0,0000 & 125,5628 & 0,0000 & 0,0000 & 0,0000 & 0,0000 & .0000 & 0,0000 & 0,0005 \\
\hline $\mathrm{NaOH}$ & 0,0000 & 0,0000 & 0,0000 & 0,0000 & 0,0000 & 0,0000 & 0,0000 & 0000 & 0,0543 & 0000 & 0,0000 & ,0000 \\
\hline $\mathrm{Na}_{2} \mathrm{SO}_{4}$ & 0,0000 & 0,0000 & 0,0000 & 0000 & 0,0000 & 0,0000 & 0,0000 & 0000 & 0,0000 & 0,0000 & 0,0000 & 0,0000 \\
\hline
\end{tabular}

A Tabela 4 mostra a lista dos principais equipamentos e suas condições de operação. Com relação às colunas, é mostrado que as mesmas condições operacionais podem ser utilizadas para processar ambos os óleos, exceto na coluna de purificação do biodiesel, onde foi preciso utilizar um estágio teórico a mais para colocar o combustível dentro das especificações.

Tabela 4 - Condições de operação dos equipamentos

\begin{tabular}{|c|c|c|c|c|c|c|c|c|}
\hline Nome do Equipamento & Soja & Dendê & me do Equipamento & Soja & Dendê & Nome do Equipamento & Soja & Dendê \\
\hline \multicolumn{3}{|c|}{ Reatores } & \multicolumn{6}{|c|}{ Colunas } \\
\hline R-ESTERIFIC & & & LAV-GLI & & & LAV-ÁGUA & & \\
\hline Tempo de Residência & $3,17 \mathrm{~h}$ & $3,17 \mathrm{~h}$ & $\mathrm{~N}^{\circ}$ Estágio Teóricos & 4 & 4 & $\mathrm{~N}^{\circ}$ Estágio Teóricos & 4 & 4 \\
\hline Conversão & $95 \%$ & $95 \%$ & Pressão Topo/Fundo (Kpa) & $190 / 200$ & $190 / 200$ & Pressão Topo/Fundo (Kpa) & $110 / 120$ & $110 / 120$ \\
\hline Temperatura/Pressão & $70^{\circ} \mathrm{C} / 400 \mathrm{Kpa}$ & ${ }^{\circ} \mathrm{C} / 400 \mathrm{Kpa}$ & Temperatura Ts & $36,87 / 38,85$ & $6,87 / 38,92$ & Temperatura T & $58,63 / 60,05$ & $58,66 / 60,05$ \\
\hline R-TRANSEST & & & SEP-ETANOL-A & & & SEP-GLI & & \\
\hline Tempo de Residência & $6,6 \mathrm{~h}$ & $8,9 \mathrm{~h}$ & $\mathrm{~N}^{\circ}$ Estágio Teóricos & 5 & 5 & iricos & 5 & 5 \\
\hline Conversão & $95 \%$ & $95 \%$ & Pressão Topo/Fur & $20 / 30$ & $20 / 30$ & Pressão Top & $40 / 50$ & $40 / 50$ \\
\hline Temperatura/Pressão & $50^{\circ} \mathrm{C} / 400 \mathrm{Kpa}$ & $50^{\circ} \mathrm{C} / 400 \mathrm{Kpa}$ & Temperatura Topo/Fundo $\left({ }^{\circ} \mathrm{C}\right)$ & $42,06 / 135,16$ & $42,10 / 111,97$ & Temperatura Topo/Fundo $\left({ }^{\circ} \mathrm{C}\right)$ & $59,39 / 81,98$ & $58,40 / 81,96$ \\
\hline R-NEUTRALIZA & & & SEP-ETANOL-B & & & SEP-BIO & & \\
\hline Tempo de Residência & $1 \mathrm{~h}$ & $1 \mathrm{~h}$ & $\mathrm{~N}^{\circ}$ Estágio & 6 & 0 & ricos & 4 & 5 \\
\hline Conversão & $100 \%$ & $100 \%$ & Pressão Topo/F & $20 / 30$ & $20 / 30$ & Pressão Topo & $10 / 20$ & $10 / 20$ \\
\hline Temperatura/Pressão & $60^{\circ} \mathrm{C} / 101,3$ & $60^{\circ} \mathrm{C} / 101,3$ & Temperatura Topo/Fundo $\left({ }^{\circ} \mathrm{C}\right)$ & $42,06 / 71,89$ & $42,05 / 71,68$ & Temperatura Topo/Fundo $\left({ }^{\circ} \mathrm{C}\right)$ & $176,02 / 300,00$ & $173,53 / 300,00$ \\
\hline
\end{tabular}

Nos reatores, os tempos de residência foram obtidos para reatores CSTR, considerando que reações em batelada de 75 minutos, a $50{ }^{\circ} \mathrm{C}$ e $1 \%$ de catalisador, alcançaram $98 \%$ de conversão para a soja e $95 \%$ de conversão para o dendê, como mostrado por Garnica (2009), e supondo cinética de segunda ordem. A taxa de reação do óleo de dendê é inferior à do óleo de soja. Isso implicará em reatores maiores ou em um maior número de reatores para o processamento do óleo de dendê. $\mathrm{O}$ mesmo procedimento foi usado para a etapa de esterificação, onde em batelada foi obtido $95 \%$ de conversão em 30 minutos (Lepper e Friesenhagen, 1986). Nesse caso não foi feita distinção entre soja e dendê por não haver informação suficiente. A cinética proposta para a esterificação foi de pseudo-primeira ordem, devido ao grande excesso de álcool.

\section{CONCLUSÃO}

A produção de biodiesel a partir do óleo de soja e de dendê foi simulada nas dimensões de uma unidade média brasileira. As propriedades termodinâmicas mais importantes dos componentes foram estimadas pelo método de Constantinou e Gani. Como resultado, apesar do maior teor de acidez do dendê, ambos os óleos foram obtidos dentro das principais normas de qualidade nacionais e internacionais. A próxima etapa do trabalho seria simular possíveis soluções para os efluentes do processo, de modo a torna-lo ainda mais sustentável. Aliado a isso, uma análise econômica poderia ser feita, de modo a se obter valores reais para a comparação entre as diferentes fontes de matéria prima e as diferentes soluções de tratamento dos efluentes. 


\section{9 a 22 de outubro de 2014 \\ Florianópolis/SC}

\section{REFERENNCIAS}

CAVALCANTE, R. M. Predição da densidade de biodiesel proveniente de diversas matérias primas. Dissertação de Mestrado em Engenharia Química. Universidade Federal do Rio de Janerio, Brasil, 2012.

CONNEMANN, J.; FISCHER, J. Biodiesel in Europe 1998: Biodiesel processing technologies. International Liquid Biofuels Congress, Brasil, 1998.

CUBAS, J. L. Neutralização da Glicerina Bruta obtida pela Transesterificação dos Óleos de Crambe, Cártamo e Soja. $7^{\circ}$ Congresso Brasileiro de Plantas Oleaginosas, Óleos, Gorduras e Biodiesel. Belo Horizonte, Brasil, 2010.

GARNICA, J. A. G. Determinação experimental dos dados cinéticos da reação de transesterificação de óleos vegetais. Dissertação de Mestrado em Engenharia Química. Universidade Estadual de Campinas, Brasil, 2009.

INNOCENTINI, M. D. M. O Processo de Produção Industrial de Biodiesel. $7^{a}$ Jornada Cientifica da UFSCar, Curso de Engenharia Química. Universidade de Ribeirão Preto, Brasil, 2008.

KREUTZER, U. R. Manufacture of fatty alcohols based on natural fats and oils. J. Am. Oil Soc. Chem., v. 61 (2), p. 343-348, 1984.

LEPPER, H.; FRIESENHAGEN, L. Process for the production of fatty acid esters of short-chain aliphatic alcohols from fats and/or oils containing free fatty acids. US Patent, 4608202, 1986.

MEHER, L. C.; VIDYA, S. D.; NAIK, S. N. Technical aspects of biodiesel production by transesterification - a review. Ren. and Sust. Energy Reviews, v. 10, p. 248-268, 2006.

OLIVEIRA, P. T. J. Produção de Biodiesel por via catalítica e indução de microondas. Dissertação de Mestrado em Engenharia Química. Instituto Politécnico de Bragança, Portugal, 2008.

POLING, B. E.; PRAUSNITZ, J. M.; O’CONNELL, J. P. The Properties of Gases and Liquids. New York: Editora McGraw-Hill, 2004.

QUELHAS, T. S. S. S. Otimização Energética na Produção de Biodiesel. Monografia em Engenharia Química. Universidade Federal do Rio de Janerio, Brasil, 2012.

REID, R. C.; PRAUSNITZ, J. M.; SHERWOOD, T. K. The Properties of Gases and Liquids. New York: Editora McGraw-Hill, 1987

WEST, A. H.; ELLIS, N.; POSARAC, D. Assessment of four Biodiesel Producion Processes using Hysys Plant. Bioresource Technology, v. 99, p. 6587-6601, 2007.

ZHANG, Y.; DUBE, M. A.; McLEAN, D. D.; KATES, M. Biodiesel production from waste cooking oil: 1. Economic assessment and sensitivity analysis. Bioresource Technology, v. 89, p. 1-16, 2003.

ZHANG, Y.; DUBE, M. A.; McLEAN, D. D.; KATES, M. Biodiesel production from waste cooking oil: 2. Economic assessment and sensitivity analysis. Bioresource Technology, v. 90, p. 229-240, 2003. 